\title{
Clinical phenotype of the recurrent 1q21.1 copy-number variant
}

\author{
Raphael Bernier, PhD', Kyle J. Steinman, MD², Beau Reilly, PhD², Arianne Stevens Wallace, PhD', \\ Elliott H. Sherr, MD', Nicholas Pojman, BS ${ }^{4}$, Heather C. Mefford, MD, PhD'5, Jennifer Gerdts, PhD', \\ Rachel Earl, BS', Ellen Hanson, PhD ${ }^{6,7}$, Robin P. Goin-Kochel, PhD ${ }^{8}$, Leandra Berry, PhD \\ Stephen Kanne, PhD ${ }^{9}$, LeeAnne Green Snyder, PhD ${ }^{6,10}$, Sarah Spence, MD ${ }^{11}$, Melissa B. Ramocki, MD ${ }^{12}$, \\ David W. Evans, PhD ${ }^{13}$, John E. Spiro, PhD ${ }^{10}$, Christa L. Martin, PhD ${ }^{13,14}$, David H. Ledbetter, $\mathrm{PhD}^{13,14}$ and \\ Wendy K. Chung, MD ${ }^{15,16}$; on behalf of the Simons VIP consortium.
}

\begin{abstract}
Purpose: To characterize the clinical phenotype of the recurrent copy-number variation (CNV) at 1q21.1, we assessed the psychiatric and medical phenotypes of 1q21.1 deletion and duplication carriers ascertained through clinical genetic testing and family member cascade testing, with particular emphasis on dimensional assessment across multiple functional domains.
\end{abstract}

Methods: Nineteen individuals with 1q21.1 deletion, 19 individuals with the duplication, and 23 familial controls (noncarrier siblings and parents) spanning early childhood through adulthood were evaluated for psychiatric, neurologic, and other medical diagnoses, and their cognitive, adaptive, language, motor, and neurologic domains were also assessed. Twenty-eight individuals with 1q21.1 CNVs (15 deletion, 13 duplication) underwent structural magnetic resonance brain imaging.

Results: Probands with 1q21.1 CNVs presented with a range of psychiatric, neurologic, and medical disorders. Deletion and

\section{INTRODUCTION}

The 1q21.1 copy-number variant (CNV), which spans $\sim 800 \mathrm{~kb}$ of unique DNA sequence and includes at least 7 genes, is associated with a highly variable phenotype. Brunetti-Pierri et al. ${ }^{1}$ and Mefford et al. ${ }^{2}$ first reported findings of 1q21.1 CNV in clinical populations. Deletions and duplications at the 1q21.1 locus are more frequent in clinical series of individuals with intellectual disability, developmental delay, schizophrenia, and autism spectrum disorder (ASD) compared with controls. ${ }^{1-7}$ Phenotypes associated with 1q21.1 also include other psychiatric diagnoses such as learning disabilities, attention-deficit hyperactivity disorder (ADHD), bipolar disorder, anxiety, and depression. ${ }^{1,3,8}$ Although both deletions and duplications have been reported in individuals with schizophrenia and ASD, deletions have been more strongly associated with schizophrenia duplication carriers shared several features, including borderline cognitive functioning, impaired fine and gross motor functioning, articulation abnormalities, and hypotonia. Increased frequency of Autism Spectrum Disorder (ASD) diagnosis, increased ASD symptom severity, and increased prevalence of macrocephaly were observed in the duplication relative to deletion carriers, whereas reciprocally increased prevalence of microcephaly was observed in the deletion carriers.

Conclusions: Individuals with 1q21.1 deletions or duplications exhibit consistent deficits on motor and cognitive functioning and abnormalities in head circumference.

Genet Med advance online publication 11 June 2015

Key Words: autism spectrum disorder; copy-number variation; developmental disability; 1q21.1 deletion; 1q21.1 duplication

than duplications $\mathrm{s}^{9-12}$ and duplications more strongly associated with $\mathrm{ASD} /$ autistic features than deletions. ${ }^{2,3,6,8}$

Most studies of 1q21.1 have reported on individuals ascertained through disease-specific series, e.g., work performed by The International Schizophrenia Consortium (2008) and Levinson et al. ${ }^{9,12}$ Studies of patients ascertained from genetics clinics ${ }^{1-3,6}$ provide a broader understanding of the full range of the clinical phenotype but are limited in the availability of standardized, dimensionally assessed behavioral and psychiatric features.

There has been limited opportunity to compare and contrast behavioral phenotypes of individuals with reciprocal deletions and duplications at the same locus. This is due, in part, to the rarity of specific CNVs limiting sample sizes, and also because of differing ascertainment approaches, rendering equivalent comparisons impossible. The detailed characterization of

\footnotetext{
${ }^{1}$ Department of Psychiatry and Behavioral Sciences, University of Washington, Seattle, Washington, USA; ${ }^{2}$ Department of Neurology, University of Washington, Seattle, Washington, USA; ${ }^{3}$ Lakeside Center for Autism, Issaquah, Washington, USA; ${ }^{4}$ Department of Neurology, University of California San Francisco, San Francisco, California, USA; ${ }^{5}$ Department of Pediatrics, University of Washington, Seattle, Washington, USA; ${ }^{6}$ Division of Developmental Medicine, Boston Children's Hospital, Boston, Massachusetts, USA; ${ }^{7}$ Harvard Medical School, Boston, Massachusetts, USA; ${ }^{8}$ Department of Pediatrics, Baylor College of Medicine, Houston, Texas, USA; ${ }^{9}$ Thompson Autism Center, University of Missouri, Columbia, Missouri, USA; ${ }^{10}$ Simons Foundation, New York, New York, USA; ${ }^{11}$ Department of Neurology, Boston Children's Hospital, Boston, Massachusetts, USA; ${ }^{12}$ Department of Neurology, Texas Children's Hospital, Houston, Texas, USA; ${ }^{13}$ Autism \& Developmental Medicine Institute, Danville, Pennsylvania, USA; ${ }^{14}$ Genomic Medicine Institute, Geisinger Health System, Danville, Pennsylvania, USA; ${ }^{15}$ Department of Pediatrics, Columbia University, New York, New York, USA; ${ }^{16}$ Department of Medicine, Columbia University, New York, New York, USA. Correspondence: Raphael Bernier (rab2@uw.edu)
} 
specific neurobehavioral profiles associated with a locus (e.g., 1q21.1) is essential for defining genotype-phenotype relationships as a first step toward designing, implementing, and measuring success of targeted interventions.

Given the variability of the phenotype described in the literature, the limited sample sizes in previous studies, and the lack of comparison cohorts with which to more tightly refine the behavioral phenotype, we ascertained a series of individuals with the 1q21.1 deletion or duplication, conducted comprehensive and standardized behavioral, neurologic, and medical history assessments, and examined the profiles of individuals with the 1q21.1 deletion in relation to individuals with the reciprocal duplication to identify shared and distinguishing features.

\section{MATERIALS AND METHODS}

\section{Participants}

Probands with the 1q21.1 CNV were identified through routine clinical testing and registered at the Simons VIP Connect website (https://SimonsVIPConnect.org). Recruitment efforts to direct families to the website included Google Ads, links from patient advocacy and social media websites, direct mailings to medical providers across the country, and through collaborations with cytogenetics laboratories. ${ }^{13}$ Those families expressing interest in participating in research provided informed consent to enroll in the study; the study was approved by the institutional review boards at each participating site. Clinical laboratory reports were reviewed by an ABMG board-certified clinical cytogeneticist (C.L.M.) to ensure that all participants had the recurrent 1q21.1 $\mathrm{CNV}$, including $800 \mathrm{~kb}$ of unique DNA sequence and at least 7 genes (chr1:146577487-147394506 GRCh37/hg19). Previous reports have used various size ranges for this $\mathrm{CNV}$, with the most commonly used being $1.35 \mathrm{Mb}$; however, these estimates include some of the flanking segmental duplication regions and are not specific to the unique DNA sequence. Individuals whose 1q21.1 $\mathrm{CNV}$ included the adjacent thrombocytopenia-absent radius region or those with additional pathogenic CNVs, other monogenic disorders, or fetal alcohol syndrome were excluded. Other family members carrying the CNV were identified by cascade genetic testing using custom-designed oligonucleotide arrays providing genome-wide coverage at $\sim 400 \mathrm{~kb}$ resolution and targeted known disease gene coverage at a resolution of $\sim 50 \mathrm{~kb}$ ( $60 \mathrm{~K}$ or 180K microarray format, Agilent Technologies, Santa Clara, CA).
Participants included 9 children and 10 additional family members with the deletion, 10 children and 9 additional family members with the duplication, 8 full-biological noncarrier siblings of the individuals with the CNV, and 15 biological noncarrier parents of the individuals with the CNV (Table 1). If more than one sibling was available, then the sibling closest in age to the carrier was invited to participate and was labeled the designated sibling. Of the deletion cohort, eight of the nine children were the initially identified proband, whereas only one adult (age 19 years, significantly impaired and living with parents) of the 10 was the initially identified proband. Among the deletion cases, three were de novo, 12 were inherited, and four had inheritance status that was not determinable due to lack of access to both biological parents. Similarly, in the duplication cohort, 8 of the 10 children were the initially identified proband. However, in the duplication cohort, only one adult (aged 18.0 years) was the proband. Of the total duplication cases, one was de novo, 13 were inherited, and five could not be determined.

For each participant, a detailed medical history was collected through interview and medical records review prior to an in-person evaluation. Standardized psychiatric, behavioral, motor, and neurologic assessments and structural brain imaging were then conducted at one of five Simons VIP sites (Harvard University, Baylor College of Medicine, University of Washington, University of California San Francisco, and Children's Hospital of Philadelphia). Because some individuals were unable to complete all measures, the number of individuals assessed for each measure is reported in the results. Cross-site standardization of psychiatric, behavioral, and motor assessments included webinar-based and in-person training, on-site demonstration of competency, cross-site reliability maintenance through monthly conference calls, periodic video-taped review of administration, and independent validation of diagnosis and measurement by trained consultants through data and video-tape review.

\section{Measures}

Medical history interview. A structured caregiver interview was conducted to collect information regarding pregnancy, prenatal, birth, developmental, and medical history for all carriers. Adapted from the Simons Simplex Collection, ${ }^{14}$ this interview was used to identify diagnosed medical disorders.

Table 1 Demographics of 1q21.1 deletion and duplication carriers and familial noncarrier controls

\begin{tabular}{|c|c|c|c|c|}
\hline 1q21.1 status & $N$ & & Gender & $\begin{array}{l}\text { Mean age in years } \\
\text { (standard deviation) }\end{array}$ \\
\hline \multirow[t]{2}{*}{ Deletion } & 19 & Child & 3 males, 6 females & $7.2(5.1)$ \\
\hline & & Adult & 4 males, 6 females & $40.7(16.8)$ \\
\hline \multirow[t]{2}{*}{ Duplication } & 19 & Child & 8 males, 2 females & $6.1(4.1)$ \\
\hline & & Adult & 3 males, 6 females & $35.2(11.2)$ \\
\hline \multirow[t]{2}{*}{ Normal 1q21.1 copy-number familial controls ${ }^{a}$} & 23 & Child & 4 males, 4 females & $5.8(4.0)$ \\
\hline & & Adult & 6 males, 9 females & $40.3(11.9)$ \\
\hline
\end{tabular}

Child = age younger than 18 years.

aFourteen familial controls are from the deletion families and nine are from duplication families. 
Medical records were collected to confirm and clarify endorsed diagnoses.

Head circumference. Standard orbital frontal circumference was measured. Standardized head circumference values were calculated using a normative population reference. ${ }^{15}$

Psychiatric, neurocognitive, motor skill, and neurologic assessment. Standardized assessments of psychiatric diagnosis, ASD-specific symptoms, and neurocognitive abilities were conducted for all participants. Standardized assessments of overall motor skills, neurologic examination findings, and prior neurologic disorder diagnoses were conducted for all carriers.

Psychiatric diagnosis. Psychiatric diagnoses were established by experienced, licensed clinicians using all available information, including records review, clinical observation, caregiver history, and consideration of all obtained measures following DSM-IVTR diagnostic criteria. ${ }^{16}$

Autism-specific symptoms. Clinicians trained to research reliability administered the Autism Diagnostic Observation Scale $(\mathrm{ADOS})^{17}$ and the Autism Diagnostic Interview-Revised. ${ }^{18} \mathrm{ASD}$ symptom severity was calculated using the Comparison Score. ${ }^{19}$

Neurocognitive functioning. Nonverbal and verbal intelligence quotient (IQ) scores in all participants were estimated using standard or ratio scores based on results from a developmentally appropriate cognitive measure such as the Mullen Scales of Early Learning, ${ }^{20}$ the Differential Abilities Scale, Second Edition, ${ }^{21}$ or the Wechsler Abbreviated Scales of Intelligence. ${ }^{22}$ The overall adaptive composite from the Vineland Adaptive Behavior Scales, Second Edition, survey interview ${ }^{23}$ was used to assess adaptive skills in the carriers and designated siblings. Phonological shortterm memory was assessed using the nonword repetition subtest from the Comprehensive Test of Phonological Processing, ${ }^{24}$ yielding a standardized score for all child participants.

Motor skills. Overall (gross and fine) motor abilities were evaluated dimensionally for CNV carriers between the ages of 3 and 17 years old using the total standard score from the Movement Assessment Battery for Children-2 (Movement ABC-2; ref. ${ }^{25}$ ). Fine motor abilities were quantified using the both-hands score from the Purdue Pegboard Test for all participants. ${ }^{26}$

Neurologic examination. A standardized neurologic examination was performed on all carriers. The examination included assessment of articulation, cranial nerve function, muscle bulk and tone, power, deep tendon reflexes, adventitial movements, cerebellar function, gait, and agility, plus examination for sacral dimples and neurocutaneous abnormalities (Table 2).

\section{Best available neurologic history (BANH)}

All available clinical neurologic records (clinic and consultation notes by neurologists, developmental pediatricians, or neurosurgeons, and head CT, brain MRI, and EEG reports) were obtained for each subject and reviewed through interviews by the neurologist. Based on this neurologist interview and neurologic record review, the site neurologist determined a best available neurologic history (BANH), which included confirmation that each sign had been diagnosed by a medical professional. If the neurologist was "not sure" whether a sign/ diagnosis had been identified, then the subject was excluded from the analysis for that item.

\section{Imaging measures}

The 1q21.1 CNV carriers and a sample of age- and gendermatched noncarrier controls completed a structural MRI scan of the brain. Noncarrier controls were individuals participating in a different imaging study in which genetic status was identified and no 1q21.1 CNVs were present. For participants who visited the University of California San Francisco, Harvard University, or Children's Hospital of Philadelphia, the structural scan was obtained using a 3-tesla TIM Trio magnetic resonance imaging (MRI) scanner, using the vendor-supplied 32-channel phasedarray radiofrequency head coils. Structural data included a high-resolution multiecho T1-weighted magnetization-prepared gradient-echo image (ME-MPRAGE; ref. 27) using the following parameters: repetition time $(\mathrm{TR})=2,530 \mathrm{~ms}$; time interval $(\mathrm{TI})=1,200 \mathrm{~ms}$; echo time $(\mathrm{TE})=1.64 \mathrm{~ms}$; flip angle $(\mathrm{FA})=7^{\circ} ; 1 \times 1 \times 1 \mathrm{~mm}$; and field of view $(\mathrm{FOV})=256 \mathrm{~mm}$. For participants examined at University of Washington and Baylor University, an equivalent structural scan was obtained using a Philips scanner with an 8-channel head coil (TR $=6.6 \mathrm{~ms}$; TE $=3 \mathrm{~ms} ; \mathrm{TI}=677.5 \mathrm{~ms} ; \mathrm{FOV}=256 \times 240 \mathrm{~mm}$; voxel size $=1 \times 1$ $\times 1 \mathrm{~mm} ; \mathrm{FA}=9^{\circ} ; 160$ slices). Three pediatric neuroradiologists naive to genetic status independently read structural MRI scans. The following structures were assessed for abnormalities in the following: corpus callosum (enlarged or reduced), pituitary, cerebellar ectopia (present or absent), cerebellar hemispheres, cerebellar vermis volume, platybasia (present or absent), dens, cortical malformations (present or absent), ventricles (enlarged or reduced), basal ganglia or thalamic abnormalities, hippocampal structure, and white matter volume. Any discrepancy was resolved by consensus review.

\section{Analytic approach}

We compiled descriptive information regarding the complete sample of 1q21.1 deletion or duplication carriers, their noncarrier parents, and designated sibling. Noncarrier parents and siblings of probands with both the deletion and duplication served as the noncarrier comparison group. To contrast individuals with the deletion to those with the duplication regarding frequency of neurologic, other medical, and psychiatric disorders, neurologic examination findings, and structural variants revealed through brain imaging, analyses included nonparametric testing as a function of event type (deletion or duplication). Univariate analyses of variance were conducted to examine dimensionally assessed domains of functioning across individuals with deletions, duplications, and familial controls. 
Table 2 Psychiatric, neurologic, and other medical problems in individuals with the 1q21 deletion or duplication

\begin{tabular}{|c|c|c|c|}
\hline & Deletion $(N=19)$ & Duplication $(N=17)$ & \\
\hline Psychiatric disorder (DSM-IV-TR code) & $N(\%)$ & $N(\%)$ & Significance (Fisher's exact) \\
\hline Anxiety and mood disorders (300.0, 300.02, 300.4, 300.9) & $5(26 \%)$ & $3(18 \%)$ & \\
\hline Behavior disorder $(312.9,313.82)$ & $0(0 \%)$ & $3(18 \%)$ & $P=0.09$ \\
\hline Autism spectrum disorder $(299.00,299.80)$ & $2(10 \%)$ & $7(41 \%)$ & $P=0.05$ \\
\hline Developmental coordination disorder (315.4) & $3(16 \%)$ & $4(23 \%)$ & \\
\hline Intellectual disability $(317,318,319)$ & $2(10 \%)$ & $5(29 \%)$ & \\
\hline Medical disorders & $N=12$ & $N=11$ & \\
\hline Cataracts & $4(33 \%)$ & 0 & \\
\hline Cardiac problem ${ }^{a}$ & $4(33 \%)$ & $3(27 \%)$ & \\
\hline Celiac disease & 0 & $1(9 \%)$ & \\
\hline Inflammatory bowel disease & $2(17 \%)$ & $1(9 \%)$ & \\
\hline Short stature & $6(50 \%)$ & $3(27 \%)$ & \\
\hline Diabetes & $1(8 \%)$ & $1(9 \%)$ & \\
\hline Recurrent infections & $1(8 \%)$ & $1(9 \%)$ & \\
\hline Scoliosis & $1(8 \%)$ & $4(36 \%)$ & \\
\hline Craniosynostosis & $1(8 \%)$ & 0 & \\
\hline Polydactyly & $1(8 \%)$ & 0 & \\
\hline Cleft palate & 0 & $1(9 \%)$ & \\
\hline Best available neurologic history diagnoses & $N=18$ & $N=19$ & \\
\hline Hypotonia & $7(39 \%)$ & $3(16 \%)$ & \\
\hline Hypertonia & $0(0 \%)$ & $0(0 \%)$ & \\
\hline Weakness & $0(0 \%)$ & $1(5 \%)$ & \\
\hline Unprovoked seizures/epilepsy & $3 / 17(18 \%)$ & $0(0 \%)$ & $P=0.1$ \\
\hline Neurologic examination findings & $N=18$ & $N=19$ & \\
\hline Sacral dimple & $2 / 17(12 \%)$ & $3(16 \%)$ & \\
\hline Articulation abnormality & $4 / 17(24 \%)$ & $6 / 16(38 \%)$ & \\
\hline Extraocular muscle weakness & $0(0 \%)$ & $2(11 \%)$ & \\
\hline Difficulty with convergence & $2 / 15(13 \%)$ & $5 / 15(33 \%)$ & \\
\hline Esotropia/exotropia & $1(6 \%$; eso) & $1(5 \% ; \mathrm{exo})$ & \\
\hline Smooth pursuit & $1(6 \%)$ & $0 / 18(0 \%)$ & \\
\hline Facial diplegia/hypotonia/drooling & $1(6 \%)$ & $2 / 16(12 \%)$ & \\
\hline Diffuse low bulk & $0(0 \%)$ & $0(0 \%)$ & \\
\hline Truncal and/or symmetric limb hypotonia & $6(33 \%)$ & $7(37 \%)$ & \\
\hline Limb weakness & $1(6 \%)^{c}$ & $0(0 \%)$ & \\
\hline Any hyporeflexia & $1 / 17(6 \%)$ & $6 / 18(33 \%)$ & $P=0.09$ \\
\hline Any hyperreflexia/clonus & $6 / 17(35 \%)$ & $5 / 18(28 \%)$ & \\
\hline Dystonia, chorea, or ataxia & $0(0 \%)$ & $0(0 \%)$ & \\
\hline Tic & $3(17 \%)$ & $0(0 \%)$ & $P=0.1$ \\
\hline Tremor & $8(44 \%)$ & $6(32 \%)$ & \\
\hline Upper or lower extremity dysrhythmia (tapping) & $2 / 16(12 \%)$ & $1 / 12(8 \%)$ & \\
\hline Dysmetria (finger-nose-finger and/or heel-knee-shin) & $0 / 16(0 \%)$ & $1 / 12(8 \%)$ & \\
\hline Agility abnormalities $^{d}$ & $3 / 17(18 \%)$ & $7 / 18(39 \%)$ & \\
\hline
\end{tabular}

aln the children, one has an arrhythmia, and three have congenital heart defects. In the adults, three have possible arrhythmias. ${ }^{b} \mathrm{Hearing}$ loss (see text). 'Mild weakness in one lower extremity. ${ }^{\mathrm{d} A b n o r m a l i t i e s ~ w i t h ~ c a s u a l ~ g a i t ~(w i d e-b a s e d, ~ w a d d l i n g, ~ s h u f f l i n g, ~ t o e-w a l k i n g, ~ o r ~ b i l a t e r a l ~ d e c r e a s e d ~ a r m ~ s w i n g) ; ~ j u m p i n g ~(~} \geq 3$ years old); hopping ( $\geq 5$ years old); standing on one foot ( $\geq 6$ years old); and/or heel, toe, or tandem ( $\geq 6$ years old) gaits. Note: significant and trending $p$ values $<1.0$ are reported above. 
In addition to the effect size and probability, differences between probands and noncarrier relatives were expressed in terms of the standard deviations of the respective dependent measures. Dependent variables included standardized assessment outcomes of interest: nonverbal IQ, verbal IQ, fine motor ability, overall adaptive ability, phonological processing ability, and ASD severity. An independent samples $t$-test was conducted to examine Movement ABC-2 scores comparing deletion and duplication carriers. To capitalize on all available data, the IQ variables included the full control sample and the remaining variables included only those individuals with available data. Finally, to examine the associations between head size and domains of functioning, correlations between standardized head circumference values and dimensionally assessed cognitive variables were calculated.

\section{RESULTS}

\section{Deletion cases}

In deletion cases, the most common psychiatric disorders included internalizing disorders, such as mood and anxiety disorders (26\%). The most commonly reported nonneurologic medical problems included short stature (50\%), cataracts (33\%), and cardiac problems (Table 2). Mean verbal and nonverbal IQ for the deletion cases were in the average range and adaptive functioning mirrored this (Table 3 ). However, when only children were considered, adaptive functioning was in the borderline range. Phonological processing, assessed only in children, was in the "extremely low" range. Fine motor performance scores for all carriers were more than 1 standard deviation below the mean of same-age peers and more than 2 standard deviations below the mean in children alone. Overall motor function scores measured in children were approximately 1 standard deviation below age norms. Mean autism severity was in the nondiagnostic range. Neurologic examination findings (Table 2) observed most frequently included tremor (44\%), hyperreflexia (35\%), and truncal and/or limb hypotonia (33\%). As identified by BANH (Table 2), the most common neurologic diagnoses included hypotonia (39\%), microcephaly (22\%), and seizures (18\%). Also seen with high frequency was hearing loss in three (17\%) deletion carriers: a 28-year-old with mild sensorineural hearing loss; a deletion carrier who developed hearing loss in her 30 s and began wearing hearing aids at age 49 years; and a deletion carrier with profound bilateral sensorineural hearing loss diagnosed at 5 months of age. Neck-tongue syndrome was suggested as a diagnosis by a nonneurologic specialist in a 13-year-old with neck pain and hemitongue numbness whose father had the same symptoms but was not diagnosed. Of the 10 individuals who had had clinical brain imaging (three MRI

Table 3 Mean scores on neuropsychometric testing.

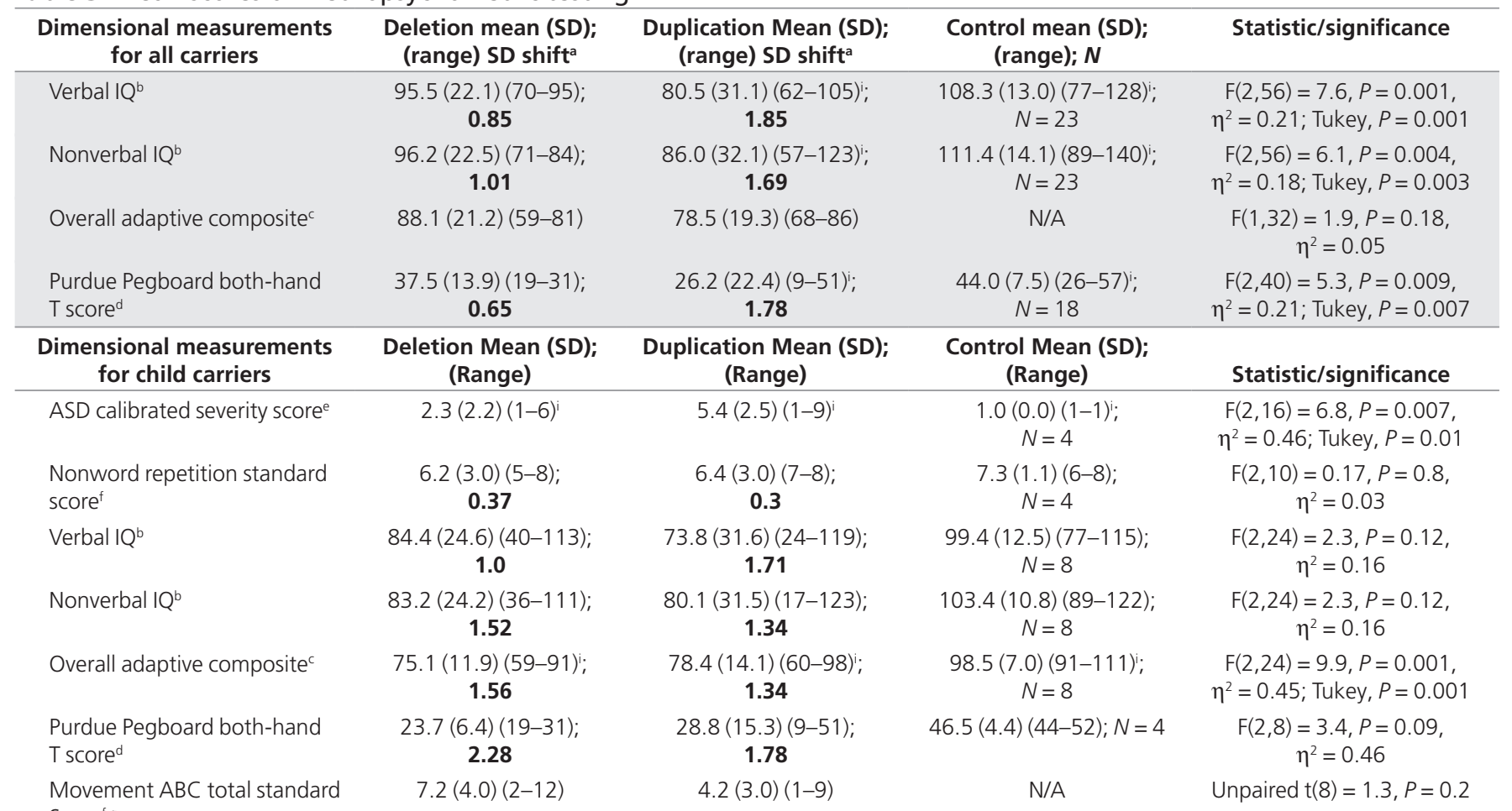

Scoref,g

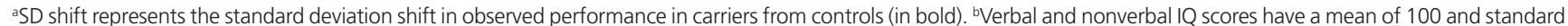
deviation of 15. 'Overall adaptive composite score has a mean of 100 and standard deviation of $15 .{ }^{\mathrm{d} P u r d u e} \mathrm{~T}$ scores have a mean of 50 and standard deviation of $10 .{ }^{e} \mathrm{Calibrated}$ severity score ranges from 1-10; scores 1-3 are in the typical range. It is based on scoring on modules 1-3 of the Autism Diagnostic Observation Schedule (ADOS). As a result, adults (who completed ADOS module 4) are not included in the comparison. ${ }^{\mathrm{f} N o n w o r d ~ r e p e t i t i o n ~ s t a n d a r d ~ s c o r e s ~ a n d ~ M o v e m e n t ~ A B C ~ T o t a l ~ S t a n d a r d ~ S c o r e s ~ h a v e ~ a ~ m e a n ~ o f ~}$ 10 and standard deviation of 3 . These were only conducted with child participants. 9Movement ABC conducted on subset of five deletion and five duplication child carriers. iPost hoc testing reveals significant difference between groups. 
only, three CT only, four both), no clinically significant findings were identified. Four individuals had undergone EEG, revealing abnormalities in three (who had all been diagnosed with seizures): one with focal sharp activity, one with focal and generalized sharp activity, and one with unspecified abnormalities.

\section{Duplication cases}

The most common psychiatric/developmental disorders observed in the duplication carriers (Table 2) included ASD (41\%), ADHD (29\%), and intellectual disability (29\%). The most commonly reported medical problems included scoliosis (36\%), short stature (27\%), and gastric ulcers (27\%). In contrast to deletion cases, mean verbal and nonverbal IQ scores for the duplication group were in the low average range, with adaptive functioning in the borderline range (Table 3). Similar to the deletion cases, phonological processing, collected only in children, was in the "extremely low" range for duplication carriers, with an average scaled score 2 standard deviations below the population mean. Fine motor impairment was observed as well, with performance falling more than 2 standard deviations below the mean of same age peers in all carriers and in children alone. Similarly, overall motor function scores measured in children was nearly 2 standard deviations below age norms. Mean autism severity fell within the ASD diagnostic range. The most commonly identified neurologic examination findings (Table 2) included abnormalities of gait/agility (39\%), articulation abnormalities (38\%), and limb and/or truncal hypotonia (37\%). On review of BANH (Table 2), the most common neurologic diagnoses were macrocephaly (26\%) and hypotonia (16\%). A 9-year-old duplication carrier used a hearing aid for unilateral sensorineural hearing loss. Seven duplication carriers had clinical brain imaging (two MRI only, two CT only, three both) with MRIs revealing the following: one individual with periventricular nodular heterotopia along the lateral aspect of a mildly dilated left ventricular atrium and occipital horn; one with 9-mm cerebellar tonsillar ectopia/ Chiari I malformation; and one 11-year-old with right greater than left posterior periventricular white matter gliosis and thinning and ex vacuo ventricular dilatation of the right posterior horn (with prenatal/postnatal course complicated only by second trimester blunt trauma). Of seven duplication cases with EEGs, only one (who did not have seizures) had an abnormal EEG (focal sharp activity and focal slowing).

\section{Comparison of deletions and duplications}

Psychiatric diagnosis. Fisher's exact test indicates the rate of ASD diagnoses was significantly higher for duplication carriers relative to the deletion carriers $(P=0.05)$, whereas a trend was observed in the frequency of ADHD diagnoses $(P=0.07)$ and behavior disorders (e.g., conduct disorder; $P=0.09$ ). When comparing only the initially identified probands, the same pattern was observed.

Medical diagnosis. No differences were observed between frequency of medical (i.e., nonneurologic) diagnoses in the modest number of patients examined. However, ANOVA revealed significant differences between $\mathrm{CNV}$ groups on mean standardized head circumference values $(\mathrm{F}(1,28)=31.9$, $\left.P<0.001, \eta^{2}=0.53\right)$. As shown in Figure 1a, head circumference for the deletion carriers (mean $\mathrm{z}$ score $=-1.5, \mathrm{SD}=1.1$ ) was significantly smaller than the duplication carriers (mean $\mathrm{z}$ score $=1.0, \mathrm{SD}=1.3$ ). This same pattern was observed in both the probands and in the adults identified through cascade genetic testing when examined separately.

Neurologic examination and BANH findings. Comparing the two cohorts revealed no significantly different neurologic

a

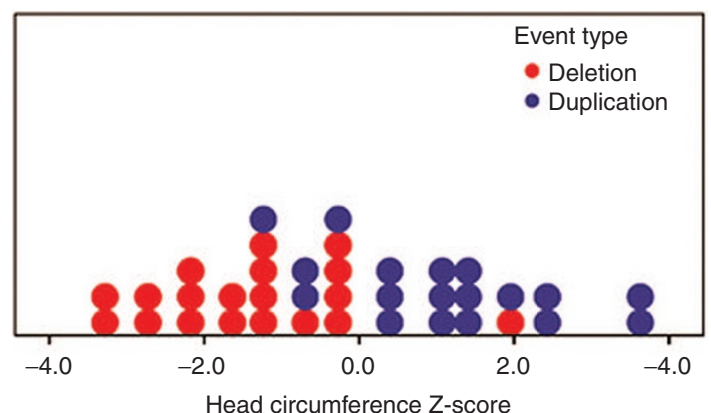

b

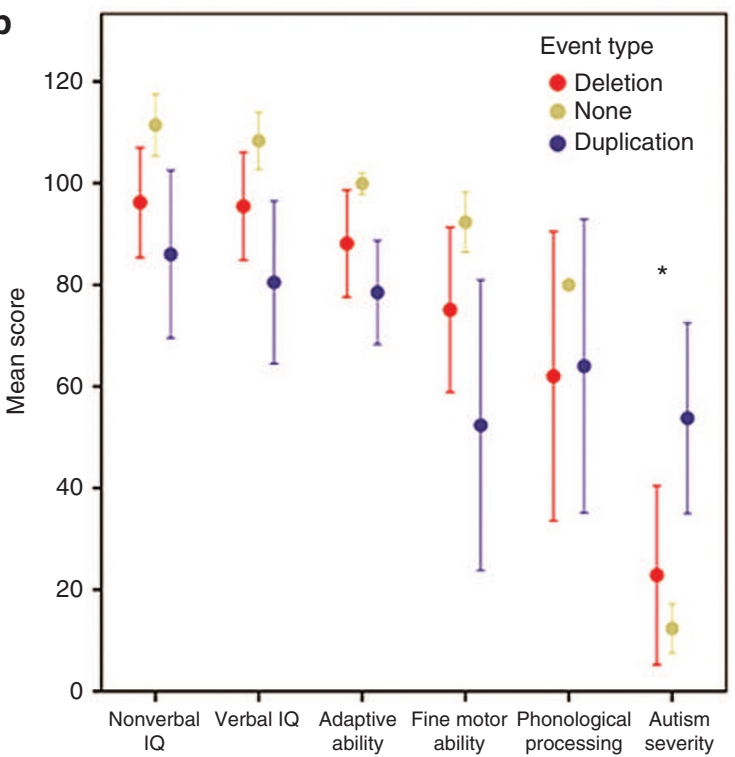

Figure 1 Dimensional Assessment of Participants. (a) Histogram revealing smaller mean standardized head circumference for deletion carriers (red) relative to duplication carriers (blue) collected during clinical site visits. Head circumference was collected from a total of 19 deletion carriers and 18 duplication carriers. (b) Mean performance across domains of functioning for individuals with deletions, individuals with duplication, and familial noncarrier controls. Cognitive ability is represented as nonverbal and verbal IQ, and adaptive functioning is reflected by an overall adaptive composite. IQ and adaptive functioning estimates have a mean of 100 and standard deviation of 15. Mean values for motor ability, defined by Purdue T scores, phonological processing, defined by a nonword repetition task, and autism severity, defined by the comparison score, have been re-scaled to a mean of 100 and standard deviation of 15. Greater comparison scores signify increased autism severity. ${ }^{*}$ Significant difference between deletion and duplication carriers. 
examination findings, although the frequency of diagnosis of macrocephaly was significantly higher in the duplication carriers $(P=0.05)$ and the frequency of diagnosis of microcephaly was significantly higher in the deletion carriers $(P=0.05)$.

Dimensional domains of functioning in all cases. As shown in Figure $\mathbf{1 b}$, no differences were observed between duplications and deletions on IQ measures; however, the duplication group performed poorer on measures of verbal IQ than the familial controls, resulting in a 1.85 SD deleterious impact, and showed significantly poorer performance in nonverbal IQ, a 1.69 SD deleterious impact (Table 3). Similarly, no differences were observed between duplication and deletion carriers on adaptive functioning, phonological processing, and fine motor abilities. However, the duplication cases scored significantly worse than familial controls in fine motor functioning. Additionally, there was increased autism symptom severity for the duplication carriers relative to the deletion carriers $(P=0.05)$.

Domains of functioning in child carriers. As shown in Figure 2, the child carriers' mean scores on nonverbal and verbal IQ fell below the adult carriers' mean scores for the deletion group $(t(17)=2.8, P=0.01$ and $t(17)=2.3, P=0.03$ for NVIQ and VIQ, respectively) but not for the duplication group $(t(15)=0.9, P=0.38$ and $t(15)=1.1, P=0.30$ for NVIQ and VIQ, respectively). Although lower IQ scores were observed when studying only the children in both cohorts, these results

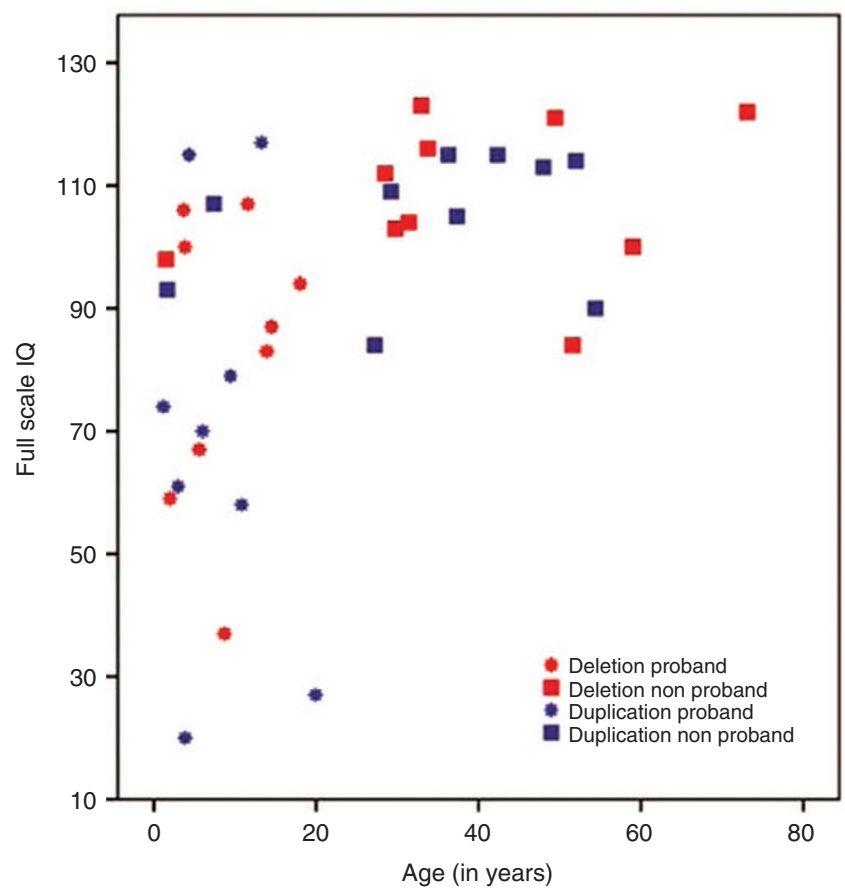

Figure 2 Dimensional assessment of participants. Relationship between overall cognitive ability, proband status, and age in the cohort showing that the proband children in both the deletion and duplication groups had a greater range of cognitive abilities that extended into the impaired range, whereas the adults, identified through cascade testing, had cognitive abilities that spanned the average to above average range. were not statistically significant when comparing children in the deletion/duplication groups relative to familial controls due to reduced power resulting from the smaller sample of children. Still, the deletion appears to confer a 1.0 SD deleterious impact on verbal IQ and a 1.52 SD impact on nonverbal IQ relative to noncarrier relatives, whereas the duplication confers a $1.74 \mathrm{SD}$ impact on verbal IQ and a 1.43 SD impact on nonverbal IQ relative to noncarrier relatives. No differences were observed between deletion and duplication carriers on overall motor functioning.

Relationship of head circumference to neurocognitive functioning. Head circumference was moderately negatively associated with fine motor functioning $(r(22)=-0.51, P=0.01)$ in both deletion and duplication carriers. Although the direction of the relationship was similar when examining deletion and duplication carriers separately, only the duplication cases remained significant $(r(9)=-0.60, P=0.05)$. A similar negative correlation was identified between head size and verbal IQ $(r(34)=-0.36, P=0.03)$ for both groups, but not with any of the other dimensionally assessed domains of functioning.

Brain imaging findings. Standardized, three-dimensional structural brain imaging was obtained from 15 deletion carriers, 13 duplication carriers, and 56 age-matched, gender-matched, noncarrier controls. Qualitative assessment demonstrated that 3 of 13 1q21.1 duplication carriers had reduced corpus callosum volume. The same 3 of 13 had concomitantly increased volumes of the lateral ventricles, and 2 of 13 had a corresponding decrease in white matter volume. Relative to frequencies of matched controls, both the enlarged ventricles and the reduced corpus callosum size remained significant after correction for multiple comparisons ( $P=0.014 ; P=0.05$, respectively). Deletion carriers did not exhibit structural differences relative to matched controls.

\section{DISCUSSION}

Comprehensive clinical examination, including dimensional assessment across several domains, revealed that psychiatric, neurologic, and medical disorders are common in individuals with both the 1q21.1 deletion and duplication. Although individuals with deletions or duplications shared several traits (e.g., borderline cognitive functioning, motor impairments, and articulation abnormalities), increased rates of ASD diagnoses and increased dimensional ASD symptom severity were observed in the duplication carriers relative to deletion carriers. Additionally, the duplication cases showed significantly greater deficits in verbal cognitive abilities and fine motor functioning relative to familial controls. Even when traditional diagnostic thresholds are not met for intellectual disability (e.g., IQ $<70$ ), our data indicate that, on average, duplication probands exhibit a 1.69 SD and 1.85 SD deleterious impact on nonverbal and verbal IQ, respectively. The observed high frequency of ASD and other associated developmental abnormalities in the duplication cases is consistent with previous findings. ${ }^{2,6,12}$ In the 
existing published reports of individuals with 1q21 CNVs, there is not a clear pattern of greater impairment for either the deletions or duplications, although our data suggest greater deficits in ASD-related domains for the duplication cases and larger deleterious impact of the duplication on cognitive ability. There is little information in the published cases regarding dimensional assessment of functioning, rendering comparison to our findings difficult. Previously reported duplication cases have similar rates as deletion cases of ASD diagnosis ${ }^{6}$ and developmental delay, ${ }^{1,2}$ suggesting, at least at the categorical level, the deleterious impact of both CNVs is sufficient to cross diagnostic thresholds and neither emerges as more impairing.

The neurologic and other medical findings in our series are similar to previous reports about 1q21.1 deletions and duplications, which indicate the increased frequency of cardiac problems, short stature, scoliosis, seizures, sensorineural hearing loss, and cataracts. ${ }^{2,8}$ The reciprocal phenotypes of microcephaly and macrocephaly have also been previously reported ${ }^{1,2}$ in clinically ascertained patients. We identified a significant inverse association between head size and both verbal IQ and fine motor ability in duplication carriers. Associations between head size and fine motor ability have inconsistently been found in other populations, ${ }^{28-31}$ but this finding highlights a moderately strong relationship between two aspects of the 1q21.1 phenotype and provides an avenue for further examination of the brain abnormalities underlying functioning in individuals with 1q21.1.

Although a range of neurodevelopmental challenges are present in the sample of clinically ascertained individuals with 1q21.1 deletion or duplication, there are commonalities of this locus compared with other CNVs associated with neurodevelopmental disorders. For example, although $80 \%$ of clinically referred individuals with $16 \mathrm{p} 11.2$ deletion present with significant neurodevelopmental disturbance, such as developmental delay or intellectual disability, and 33\% met criteria for an ASD diagnosis, recurrent language impairment is also particularly notable in this population..$^{32}$ As another example, Stefansson et al. ${ }^{33}$ compared neurocognitive traits and learning difficulties of individual neuropsychiatric CNVs, other CNV controls, clinically referred patients diagnosed with schizophrenia, and population controls, and found that cognitive deficits were common but varied in presentation across CNVs. After adjusting for IQ, individuals carrying the 15q11.2 deletion had a history of dyslexia and dyscalculia and only modest effects on other cognitive traits. With this clinically referred sample of individuals with the 1q21.1 CNV, a pattern of subtle, but consistent, fine motor impairment was observed along with neurobehavioral impairments marked by greater impairment in the duplication carriers.

Given the results of our study, the medical evaluation of individuals with 1q21.1 deletions/duplications should include evaluation for psychiatric, neurologic, and other medical concerns including autism spectrum disorder, intellectual disability, attention-deficit hyperactivity disorder, sensorineural hearing impairments (observed as young as infancy), seizures, cardiac disease, and motor difficulties (including fine and gross motor function, articulation, and eye movements). Also important to keep in mind are potential increased frequencies of tics and tremors, which were noted on examination and by families but had not been commonly diagnosed. Additionally, imaging results demonstrate enlarged ventricles and reduced corpus callosum size for some duplication cases but not deletion cases. Finally, given the frequency of mood and anxiety disorders in adults in this sample, adults should be screened for psychiatric symptoms. Given that these psychiatric disorders were not identified in the children with either deletions or duplications, it is plausible that the mood and anxiety disorders are, in part, correlated with family functioning when family members have significant psychiatric or medical concerns. Alternative hypotheses are that anxiety and mood disturbances develop over time and/or that increased awareness of subtle, but present, underlying cognitive and psychiatric difficulties associated with 1q21 CNVs lead to anxiety and mood problems.

More detailed examination of motor abilities is warranted given the significant decrement in motor abilities observed in both the probands and carrier family members. This novel finding of dimensionally assessed motor impairment is reflected in the frequency of developmental coordination disorder identified through our evaluation. Almost one-quarter of the duplication carriers and $16 \%$ of the deletion carriers met diagnostic criteria for developmental coordination disorder. Although motor challenges meeting criteria for developmental coordination disorder were not observed in the adult carriers, challenges in fine motor precision and on neurologic examination suggest a contribution of the 1q21.1 locus for fine motor ability even into adulthood. Our finding of motor impairment in adults without neuropsychiatric disorders expands on the clinical reports of motor delay in the 1q21.1 literature. ${ }^{34}$

In summary, in this carefully examined and comprehensively phenotyped sample of individuals with the same 1q21.1 deletion or duplication, we identified a high frequency of ASD and motor impairments in 1q21.1 duplication carriers. We also found a persistent decrease in motor abilities in carrier adults without other neurodevelopmental disorders.

\section{ACKNOWLEDGMENTS}

This work was supported by two grants from the Simons Foundation (SFARI award 198677 to R.B., E.H., R.G.K., and W.K.C., and SFARI award 312100 to C.L.M., D.H.L.) and a grant from the National Institutes of Health (MH074090 to D.H.L. and C.L.M.). We are grateful to all the families at the participating Simons Variation in Individuals Project (Simons VIP) sites, as well as the Simons VIP working group (Simons VIP consortium, Neuron, 73:10631067, 2012). We appreciate access to phenotypic data on SFARI Base. Approved researchers can obtain the Simons VIP population dataset described in this study by applying at https://base.sfari. org. The Simons VIP Consortium includes: H Alupay, B Aaronson, S Ackerman, K Ankenmann, C Atwell, E Aylward, A Beaudet, M Benedetti, J Berman, R Bernier, A Bibb, L Blaskey, C Brewton, R Buckner, P Bukshpun, J Burko, B Cerban, Q Chen, M Cheong, 
Z Chu, W Chung, C Dale, A Dempsey, J Elgin, J Olson, Y Evans, WA Faucett, G Fischbach, S Garza, J Gerdts, S Gobuty, R GoinKochel, PE Grant, L Green Snyder, M Greenup, E Hanson, K Hines, L Hinkley, J Hunter, R Jeremy, K Johnson, S Kanne, S Kessler, S Khan, A Laakman, M Lasala, D Ledbetter, H Lee, C Lese Martin, A Lian Cavanagh, A Llorens, T Luks, E Marco, A Martin, G Marzano, K McGovern, R McNally Keehn, D Miller, F Miller, T Moss, P Mukherjee, S Nagarajan, K Nowell, J Owen, A Paal, A Packer, P Page, B Paul, N Pojman, M Proud, S Qasmieh, M Ramocki, B Reilly, T Roberts, D Shaw, E Sherr, T Sinha, B Smith-Packard, A Snow, S Spence, J Spiro, K Steinman, A Stevens, V Swarnakar, J Tjernagel, C Triantafallou, R Vaughan, N Visyak, M Wakahiro, T Ward, and J Wenegrat.

\section{DISCLOSURE}

The authors declare no conflict of interest.

\section{REFERENCES}

1. Brunetti-Pierri N, Berg JS, Scaglia F, et al. Recurrent reciprocal 1q21.1 deletions and duplications associated with microcephaly or macrocephaly and developmental and behavioral abnormalities. Nat Genet 2008;40: 1466-1471.

2. Mefford HC, Sharp AJ, Baker C, et al. Recurrent rearrangements of chromosome 1q21.1 and variable pediatric phenotypes. NEng/J Med 2008;359:1685-1699.

3. Girirajan S, Rosenfeld JA, Coe BP, et al. Phenotypic heterogeneity of genomic disorders and rare copy-number variants. N Engl I Med 2012;367:1321-1331.

4. The International Schizophrenia Consortium. Rare chromosomal deletions and duplications increase risk of schizophrenia. Nature 2008;455:237-241.

5. Levinson DF, Shi J, Wang K, et al.; Schizophrenia Psychiatric GWAS Consortium. Genome-wide association study of multiplex schizophrenia pedigrees. Am J Psychiatry 2012;169:963-973.

6. Rosenfeld JA, Traylor RN, Schaefer GB, et al.; 1q21.1 Study Group. Proximal microdeletions and microduplications of 1q21.1 contribute to variable abnormal phenotypes. Eur J Hum Genet 2012;20:754-761.

7. Moreno-De-Luca D, Sanders SJ, Willsey AJ, et al. Using large clinical data sets to infer pathogenicity for rare copy number variants in autism cohorts. Mol Psychiatry 2013;18:1090-1095.

8. Dolcetti A, Silversides CK, Marshall CR, et al. 1q21.1 Microduplication expression in adults. Genet Med 2013;15:282-289.

9. Crespi BJ, Crofts HJ. Association testing of copy number variants in schizophrenia and autism spectrum disorders. J Neurodev Disord 2012;4:15.

10. Van Den Bossche MJ, Johnstone M, Strazisar M, et al. Rare copy number variants in neuropsychiatric disorders: Specific phenotype or not? Am J Med Genet B Neuropsychiatr Genet 2012;159B:812-822.

11. Rees $E$, Walters JT, Georgieva L, et al. Analysis of copy number variations at 15 schizophrenia-associated loci. Br J Psychiatry 2014;204:108-114.

12. Levinson DF, Duan J, Oh S, et al. Copy number variants in schizophrenia: confirmation of five previous findings and new evidence for $3 q 29$ microdeletions and VIPR2 duplications. Am J Psychiatry 2011;168:302-316.
13. Simons VIP Consortium. Simons Variation in Individuals Project (Simons VIP): a genetics-first approach to studying autism spectrum and related neurodevelopmental disorders. Neuron 2012;73:1063-1067.

14. Fischbach GD, Lord C. The Simons Simplex Collection: a resource for identification of autism genetic risk factors. Neuron 2010;68:192-195.

15. Roche AF, Mukherjee D, Guo SM, Moore WM. Head circumference reference data: birth to 18 years. Pediatrics 1987;79:706-712.

16. American Psychiatric Association. Diagnostic and Statistical Manual of Mental Disorders, Fourth Edition, Text Revision (DSM-IV-TR). Washington, DC: American Psychiatric Association, 2000.

17. Lord C, Risi S, Lambrecht L, et al. The autism diagnostic observation schedulegeneric: a standard measure of social and communication deficits associated with the spectrum of autism. J Autism Dev Disord 2000;30:205-223.

18. Lord C, Rutter M, Le Couteur A. Autism Diagnostic Interview-Revised: a revised version of a diagnostic interview for caregivers of individuals with possible pervasive developmental disorders. J Autism Dev Disord 1994;24:659-685.

19. Gotham K, Pickles A, Lord C. Standardizing ADOS scores for a measure of severity in autism spectrum disorders. J Autism Dev Disord 2009;39:693-705.

20. Mullen E. Mullen Scales of Early Learning: AGS Edition. Circle Pines, MN: American Guidance Service, 1995.

21. Elliott CD. Differential Ability Scales, 2nd edition. San Antonio, TX: Harcourt Assessment, 2007.

22. Wechsler D. Wechsler Abbreviated Scales of Intelligence. San Antonio, TX: PsychCorp, 1999.

23. Sparrow SS, Cicchetti DV, Balla DA. Vineland Adaptive Behavior Scales, 2nd edition. Minneapolis, MN: NCS Pearson, Inc., 2005.

24. Wagner RK, Torgesen JK, Rashotte CA. Comprehensive Test of Phonological Processing. Austin, TX: PRO-ED, 1999

25. Henderson SE, Sugden DA, Barnett AL. Movement Assessment Battery for Children-2: Movement ABC-2: Examiner's Manual. Pearson: San Antonio, TX 2007.

26. Tiffin J. Purdue Pegboard Examiner's Manual. Chicago, IL: Science Research Associates, 1968

27. van der Kouwe AJ, Benner T, Salat DH, Fischl B. Brain morphometry with multiecho MPRAGE. Neuroimage 2008;40:559-569.

28. Harris SR. Congenital idiopathic microcephaly in an infant: congruence of head size with developmental motor delay. Dev Neurorehabil 2013;16:129-132.

29. Ireland PJ, Ware RS, Donaghey S, et al. The effect of height, weight and head circumference on gross motor development in achondroplasia. J Paediatr Child Health 2013;49:E122-E127.

30. Kuban KC, Allred EN, O'Shea TM, et al. Developmental correlates of head circumference at birth and two years in a cohort of extremely low gestational age newborns. J Pediatr 2009;155:344-9.e1.

31. Hebestreit H, Schrank W, Schrod L, Strassburg HM, Kriemler S. Head size and motor performance in children born prematurely. Med Sci Sports Exerc 2003;35:914-922.

32. Hanson E, Nasir RH, Fong A, et al.; 16p11.2 Study Group Clinicians. Cognitive and behavioral characterization of 16p11.2 deletion syndrome. J Dev Behav Pediatr 2010;31:649-657.

33. Stefansson H, Meyer-Lindenberg A, Steinberg S, et al. CNVs conferring risk of autism or schizophrenia affect cognition in controls. Nature 2014;505: 361-366.

34. Basel-Vanagaite L, Goldberg-Stern H, Mimouni-Bloch A, Shkalim V, Böhm D, Kohlhase J. An emerging 1q21.1 deletion-associated neurodevelopmental phenotype. J Child Neurol 2011;26:113-116. 